\title{
Comparación de la estructura comunitaria entre plataformas y bolones del intermareal en isla Navarino, Reserva de Biósfera Cabo de Hornos
}

\author{
Comparison of the community structures of intertidal platforms and \\ boulders in Navarino Island, Cape Horn Biosphere Reserve
}

Jaime Ojeda ${ }^{1}$, Juan Pablo Rodríguez ${ }^{1}$, Sebastián Rosenfeld ${ }^{1}$ \& Nicolás Vega ${ }^{1}$

\section{Resumen}

La zona intermareal de canales subantárticos de Magallanes presenta diversos tipos de sustratos rocosos, los cuales pueden provocar cambios en la estructura de las comunidades que habitan este ecosistema. Este estudio comparó la composición taxonómica, rasgos funcionales (e.g., móviles y sésiles) y parámetros de estructura comunitaria como riqueza, abundancia, disimilitud en dos tipos de sustratos rocosos (bolones y plataformas) durante invierno y verano en sitios del intermareal bajo en isla Navarino, canal Beagle. La riqueza de taxones fue evaluada mediante la categorización de organismos sésiles y móviles, determinando su abundancia y disimilitud entre estaciones y sustratos. Se identificaron 33 taxones. La mayor riqueza de taxones fue observada en el sustrato de bolones. El patelogasterópodo Nacella spp presentó la mayor contribución en abundancia para organismos móviles en ambos sustratos. En cuanto a la estructura comunitaria, el mayor promedio de disimilitud entre bolones y plataformas fue para organismos móviles. Los taxones que más contribuyeron a esta disimilitud fueron los carnívoros Anasterias antarctica (18\%) y Trophon geversianus (13\%). La primera especie más abundante en bolones y la segunda en plataformas. El tipo de sustrato puede afectar la estructura comunitaria, la cual se ve reflejada principalmente en la identidad, historia de vida y los rasgos morfológicos de los organismos móviles en las costas rocosas de los canales subantárticos de la Reserva de Biósfera Cabo de Hornos.

\section{Palabras Clave:}

sub-antártico, estacional, rasgos funcionales, identidad, Nacella.

\section{Abstract}

Magellanic Subantarctic channels' coastal zones have several types of rocky substrate, which might provoke changes in communities' structure. The present study compared the composition, functional traits and community structure of the low intertidal on two types of rocky substrates (boulders and platforms) during winter and summer on Navarino Island, Beagle Channel. Taxa richness was evaluated by the categorization of sessile and mobile organisms, determining their abundance and dissimilarity between seasons and type of rocky substrates. 33 taxa were identified. The highest taxa richness was found in the boulders substrate. The patellogastropod Nacella spp. had the greatest contribution to abundance for mobiles invertebrate in both substrates. The highest average dissimilarity between boulders and platforms observed for mobile organisms. The taxa that contributed most to this dissimilarity were the carnivorous Anasterias antarctica (18\%) and Trophon geversianus (13\%). The first was most abundant in boulders and the second in platforms. The type of substrate may affect the identity, life history and morphological features of mobile organisms on the rocky shores

\footnotetext{
Laboratorio de Macroalgas Antárticas y Subantárticas (LMAS), Casilla 113-D, Universidad de Magallanes,

Punta Arenas, Chile.

$\square$ jaimeojedavillarroel@yahoo.es
} 
of the Magellanic sub-Antarctic channels.

\section{Key words:}

Subantarctic, Seasonal, Functional Traits, Identity, Nacella.

\section{INTRODUCCIÓN}

La biodiversidad del intermareal rocoso esta regulada por diversos factores ambientales $y$ biológicos, que varían dependiendo de la escala espacial o temporal (Benedetti-Cecchi et al. 2001). Por ejemplo, Cruz-Motta et al. (2010) propone que la variación latitudinal de los patrones espaciales de ensambles asociados al intermareal rocoso está principalmente influenciado por la temperatura superficial del mar, precipitación y fotoperiodo. Por otro lado, si disminuimos la escala de resolución espacial, otros factores pueden ser más importantes en los cambios de la estructura comunitaria de los intermareales rocosos. Por ejemplo, cambios estacionales de la intensidad luz fotosintéticamente activa generan variaciones en la biomasa y riqueza de macroalgas intermareales (Gunnarsson \& Ingolfsson, 1995). A su vez, cambios locales como la intensidad de oleaje pueden tener efectos en la diversidad de biotopos intermareales de un sistema de fiordos (Soto et al. 2012). También, existen factores aún más locales en términos espaciales como la geomorfología costera, la orientación de la costa, tipo o tamaño del sustrato rocoso, los cuales pueden interactuar con la composición, estructura y atributos funcionales de la biodiversidad intermareal (e.g. Sousa, 1979; Gedan et al. 2011; Ramos et al. 2015).

La línea costera de los canales y fiordos subantárticos del sur de Chile está formada principalmente por afloramientos rocosos (plataformas y acantilados) que se alternan con otras costas formadas por material fragmentado no consolidado (playas de bolones) (Hervé et al. 2009). Estos hábitats rocosos tienen una alta diversidad de biotopos, probablemente asociados a factores holográficos, geológicos, oceanográficos y climáticos (Soto et al. 2012). Incluso, se propone que no existe un patrón general de zonación intermareal para las costas del estrecho de Magallanes (Benedetti-Cecchi \& Cinelli, 1997).

En altas latitudes como la ecorregión de los canales y fiordos subantárticos de Magallanes (Spalding et al. 2007) se han documentado varios ejemplos sobre cambios en diversidad beta en taxones que estructuran las zonas costeras rocosas, es decir, variaciones en la estructura comunitaria entre sitios. Por ejemplo, Rosenfeld et al. (2018) observaron cambios significativos en estructura e incluso en los grupos funcionales de macroalgas entre playas rocosas separadas por $100 \mathrm{~km}$ de distancia. A su vez, Ríos et al. (2007) en comunidades de macroinvertebrados que habitan grampones de Macrocystis pyrifera registraron una alta heterogeneidad entre sitios del estrecho de Magallanes. Otro caso es descrito por Ojeda et al. (2014), quienes describieron que en canal Beagle existen variación de diversidad beta entre comunidades de moluscos que habitan sitios intermareales distanciados por sólo cientos de metros. Estos variaciones de diversidad beta pueden generarse por varios factores, uno de ellos es el tipo de sustrato. Existe evidencia que cambios en el tipo de sustrato entre bolones y plataformas intermareales pueden generar diferencias aspectos reproductivos de una misma especie (Sherman et al. 2007), variaciones de abundancia poblacional (Dunmore \& Schiel, 2003) o cambios en los hábitos tróficos en las comunidades intermareales (Paine, 1980). Una de los efectos de estas diferencias de sustrato es que los individuos o las comunidades responden de manera diferente a los gradientes de disturbios que enfrentan estos tipos de sustratos (Sherman et al. 2007). De hecho, las diferencias en la estructura comunitaria pueden ser mayores en escalas de resolución espacial fina, es decir, centímetros o pocos metros entre unidades de muestreo (e.g. Valdivia et al. 2014). Muchas veces estas variaciones en la estructura comunitaria no son tomadas en cuenta en las metodologías conservación ecológica, pero parecen ser muy relevantes a la hora de planificaciones de reservas y parques marino-costeros (e.g., Banks \& Skilleter, 2007). Considerando la importancia de analizar una escala fina de resolución espacial de las comunidades intermareales rocosas en canales y fiordos subantárticos del sur de Chile, este trabajo propone comparar la riqueza de taxones, la estructura comunitaria y los atributos funcionales en sustratos de bolones y plataformas intermareales de la costa norte de isla Navarino, Reserva de 
Biósfera Cabo de Hornos. Finalmente, este trabajo tiene el propósito de contribuir al conocimiento de la ecología intermareal en sitios prioritarios para la conservación subantártica, como el Parque Etnobotánico Omora (Rozzi et al. 2010).

\section{MATERIALES Y MÉTODOS}

El presente estudio se realizó en bahía Róbalo y Caleta Paula, canal Beagle (545'ㅇ 67³9'O), ubicada en la costa norte de Isla Navarino, Chile (Fig. 1). Los sitios de estudio se encuentran próximos al Parque Etnobotánico Omora perteneciente a la
Red Chilena de Sitios de Estudios Socioecológicos a Largo Plazo (Rozzi et al. 2012). El trabajo de campo se realizó en el intermareal bajo de bahía Róbalo en sustratos bolones y en caleta Paula con sustratos de plataformas, durante invierno (agosto de 2011) y verano (enero de 2012). Ambas zonas tienen una orientación hacia el oeste del canal Beagle. Los sitios están distanciados por 2,8 km. En cada zona se utilizaron 15 cuadrantes al azar con una dimensión de $50 \times 50 \mathrm{~cm}$. El diseño de muestreo fue 2 (estaciones) x 2 (tipos de sustrato) $\mathrm{x}$ 15 cuadrantes $=60$ muestras (Fig. 1). Cabe destacar, que el sitio de bolones está compuesto por un 4
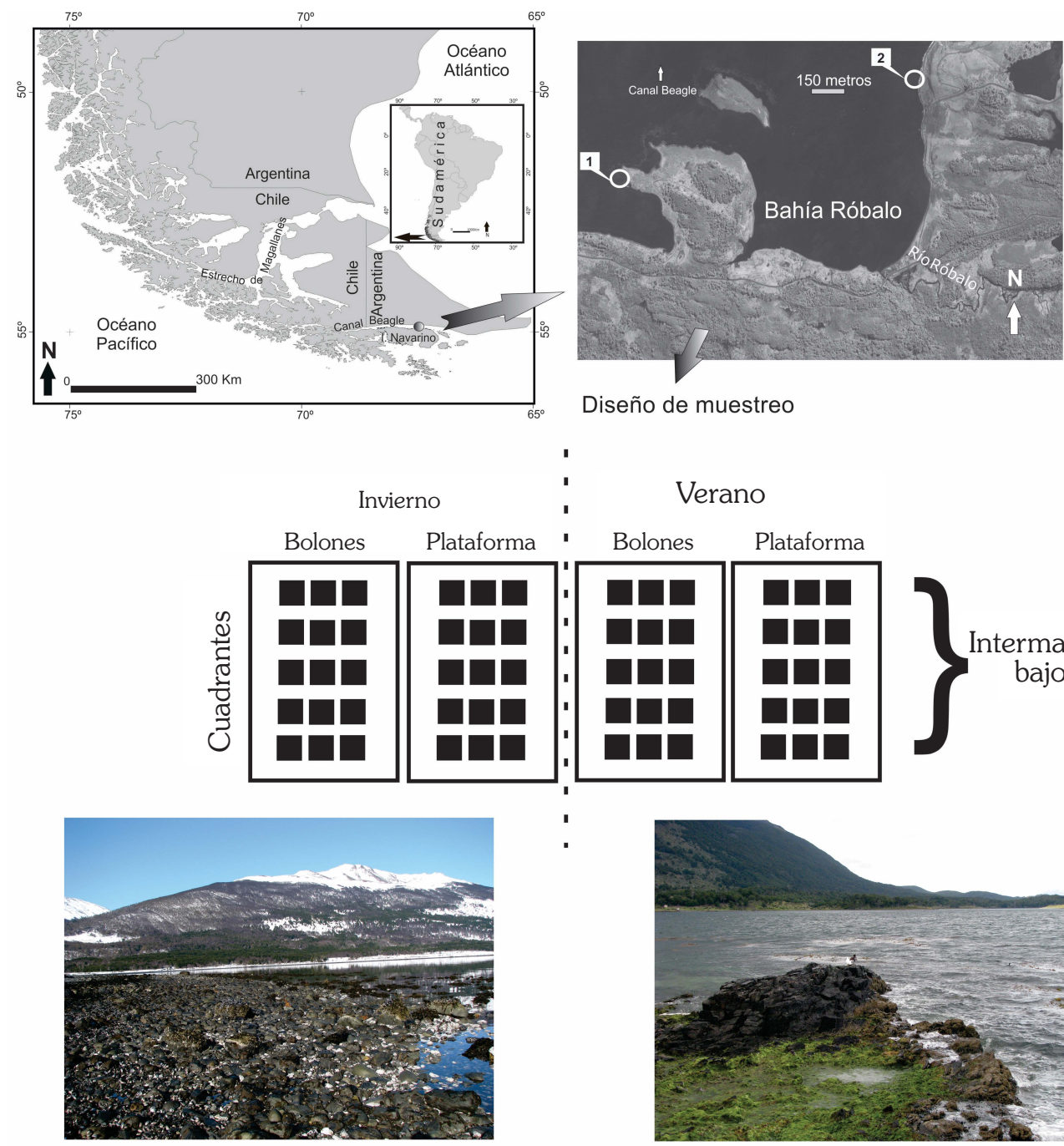

Bolones, bahía Róbalo

Verano

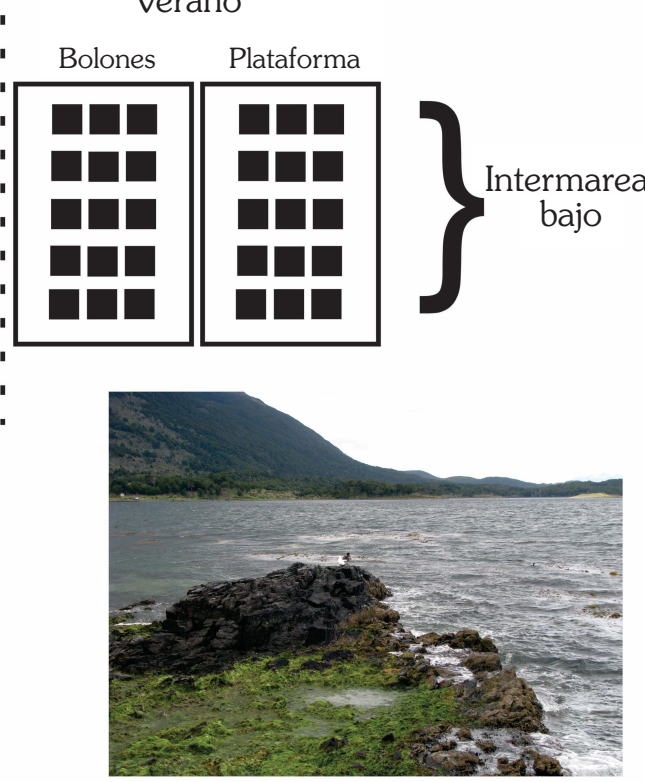

Plataforma, caleta Paula

Fig. 1. Sitio de estudio y diseño de muestreo en intermareal de Caleta Paula ( 1 =plataformas) y Bahía Róbalo ( 2 =bolones) en isla Navarino, Chile. 
\% de guijarros, $29 \%$ de canto rodado pequeño y $65 \%$ de canto rodado grande (Ojeda et al. 2014). La biota de cada cuadrante fue registrada con un fotocuadrante a una altura fija de $1 \mathrm{~m}$ de distancia del sustrato. En la zona de bolones, se contabilizaron in situ los invertebrados que habitaban bajo bolones dentro del área del cuadrante, atributo importante por tridimensionalidad de este hábitat. No fueron contabilizados organismos de la infauna e intersticios (e.g. Amphipoda e Isopoda). La razón fue su pequeño tamaño y alta movilidad, lo cual no permite estimar con precisión su abundancia en el campo. La identificación de los organismos fue estimada hasta la resolución taxonómica más específica (e.g. familia, género o especie), con el soporte de literatura taxonómica especializada para la ecorregión de subantártica de Magallanes. Por ejemplo, para la clase Polyplacophora, Gastropoda y Bivalvia se utilizó principalmente: Powell (1951), Dell (1964), Reid y Osorio (2000), Sirenko (2006), Aldea y Rosenfeld (2011). Los taxones fueron caracterizados según sus atributos funcionales, tales como: la movilidad (sésil o móvil), hábitos tróficos (filtrador, herbívoro o carnívoro) (e.g. Ojeda et al. 2014). En macroalgas se utilizó su hábito morfológico (crustósa calcárea, filamentosa, laminar, foliosa, tubular cartilaginoso) (e.g. Rosenfeld et al. 2018). Finalmente, en cada cuadrante se determinó la abundancia del tipo de cobertura, que fue medida por el porcentaje de cobertura de taxones sésiles y roca descubierta. A su vez, se determinó la abundancia de los taxones móviles fue mediante el número de individuos por taxón.

Se estimaron las diferencias en la estructura comunitaria entre bolones y plataformas mediante dos factores (porcentaje de cobertura y número de individuos), los cuales fueron comparados individualmente para ambos tipos de sustrato: i) cobertura, en donde se consideró el porcentaje de cobertura de taxones sésiles y porcentaje de cobertura de roca descubierta; y ii) taxones móviles, número de individuos por taxón. Estas comparaciones se realizaron mediante el análisis de permutaciones para agrupaciones multivariantes (PERMANOVA). Para ello, se calculó la distancia de disimilitud de Bray-Curtis entre pares de observaciones, los datos fueron transformados a raíz cuarta (para reducir la contribución de las especies abundantes y equiparar a las especies raras) y se usaron 9999 permutaciones sin restricciones de datos. Para cada efecto significativo se realizó una prueba a posteriori entre pares de grupos (pair-wise) (Anderson, 2001). Previamente, se realizó el análisis de homogeneidad de varianza PERMDISP, el cual mide la dispersión multivariante (varianza) para un grupo de muestras (Anderson, 2006). La estimación del aporte específico de los taxones a la estructura comunitaria se realizó mediante el análisis SIMPER (Clarke, 1993). Para ello, se determinó el porcentaje de contribución y el promedio de disimilitud para tipo de cobertura y taxones móviles, y se comparó entre bolones y plataformas para una misma estación (i.e., invierno o verano). Los datos fueron transformados a raíz cuarta, el porcentaje de corte tuvo un límite de $90 \%$ de contribución al componente, los datos fueron analizados con el programa PRIMER 6 (PRIMER-E, Ltd. Plymouth, UK).

\section{RESULTADOS}

\section{Riqueza taxonómica}

Se identificaron un total de 33 taxones considerando ambos tipos de sustratos rocosos. La mayor riqueza se observó en el sustrato de bolones con 26 taxones, de los cuales, 12 taxones se encontraron exclusivamente en este tipo de sustrato (Tabla 1). Mientras que en plataformas se observaron 20 taxones y 7 de ellos se encontraron sólo en este tipo de sustrato (Tabla 1).

\section{Estructura comunitaria}

El análisis multivariado de homogeneidad de varianza PERMDISP mostró que el tipo de cobertura fue homogéneo para cada tipo de sustrato rocosos $(P>0,05)$. Por otro lado, el análisis PERMANOVA que comparó la estructura del tipo de cobertura entre bolones y plataformas mostró diferencias significativa $(P<0,05)$ tanto en invierno como en verano. El análisis SIMPER mostró que el mayor promedio de disimilitud para el tipo de cobertura entre bolones y plataformas fue $56 \%$ en invierno (Fig. 2). El tipo de cobertura que más contribuyó al promedio de disimilitud en invierno y verano fueron los filtradores sésiles 
Tabla 1. Taxones encontrados en bolones y plataformas de isla Navarino, considerando su atributo funcional y su presencia en cada tipo de sustrato. $\mathrm{B}=$ bolones; $\mathrm{P}=$ plataformas; $\mathrm{B} / \mathrm{P}=$ Presencia en ambos sustratos.

\begin{tabular}{|c|c|c|}
\hline Tipo de cobertura & Atributo Funcional & Sustrato \\
\hline Roca descubierta & & $\mathrm{B} / \mathrm{P}$ \\
\hline \multicolumn{3}{|l|}{ Rhodophyta (Phylum) } \\
\hline Lithophyllum subantarcticum (Foslie, 1907) & crustosa calcárea & $P$ \\
\hline Nothogenia fastigiata (Bory) P.G.Parkinson 1983 & tubular cartilaginoso & $\mathrm{P}$ \\
\hline \multicolumn{3}{|l|}{ Ochrophyta (Phylum) } \\
\hline Caepidium antarcticum (J.Agardh, 1859) & crustosa y filamentosa & $\mathrm{B} / \mathrm{P}$ \\
\hline Lessonia flavicans (Bory, 1825) & laminar & B \\
\hline \multicolumn{3}{|l|}{ Chlorophyta (Phylum) } \\
\hline Ulva spp. & foliosa & $\mathrm{B} / \mathrm{P}$ \\
\hline \multicolumn{3}{|l|}{ Bivalvia (Clase) } \\
\hline Mytilus platensis (d'Orbigny 1842) & sésil filtrador & $\mathrm{B} / \mathrm{P}$ \\
\hline Perumytilus purpuratus (Lamarck, 1819) & sésil filtrador & $\mathrm{B} / \mathrm{P}$ \\
\hline Hiatella sp. & filtrador & $\mathrm{P}$ \\
\hline Aulacomya atra (Molina, 1782) & sésil filtrador & $\mathrm{B} / \mathrm{P}$ \\
\hline \multicolumn{3}{|l|}{ Hexanauplia (Clase) } \\
\hline Notochthamalus scabrosus (Darwin, 1854) & sésil filtrador & $\mathrm{B} / \mathrm{P}$ \\
\hline
\end{tabular}

Especies Móviles

Polyplacophora (Clase)

Ischnochiton stramineus (Sowerby, 1832)

Plaxiphora aurata (Spalowsky, 1795)

Tonicia spp.

herbivoro $\quad \mathrm{B} / \mathrm{P}$

\section{Gastropoda (Clase)}

Fissurella spp.

herbivoro

$\mathrm{B} / \mathrm{P}$

Nacella spp.

Scurria ceciliana (Orbigny, 1841)

Margarella violacea (King \& Broderip, 1832)

herbivoro $\quad \mathrm{B} / \mathrm{P}$

herbivoro $\quad \mathrm{B} / \mathrm{P}$

Pareuthria fuscata (Bruguière, 1789)

Trophon geversianus (Pallas, 1774)

herbivoro $\quad \mathrm{B}$

Acanthina monodon (Pallas, 1774)

Xymenopsis muriciformis (King, 1832)

Siphonaria lessonii (Blainville, 1824)

herbivoro

B

carroñero

B

Opisthobranchia indet.

carnívoro

$\mathrm{B} / \mathrm{P}$

carnivoro

$\mathrm{P}$

carnivoro

$\mathrm{B} / \mathrm{P}$

herbivoro

$$
\mathrm{P}
$$

$B / P$

Polychaeta indet. 
(continuación Tabla 1)

Malacostraca (Clase)

Halicarcinus planatus (Fabricius, 1775)

carroñero

B

Asteroidea (Clase)

Anasterias antarctica (Lütken, 1857)

carnívoro

B

Asterina fimbriata (Perrier, 1875)

carnívoro

Asteroidea indet.

Ophiuroidea (Clase)

Ophiuroidea indet.

Echinoidea (Clase)

Arbacia dufresnii (Blainville, 1825)

herbívoro $P$

Pseudechinus magellanicus (Philippi, 1857)

herbivoro $\mathrm{B} / \mathrm{P}$

Actinopterygii (Clase)

Harpagifer bispinis (Forster, 1801)

carnívoro B

Patagonotothen sp.

carnívoro

B

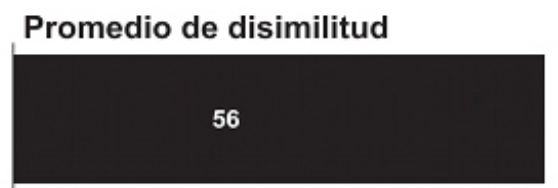

Invierno

Contribución de los componentes a la disimilitud

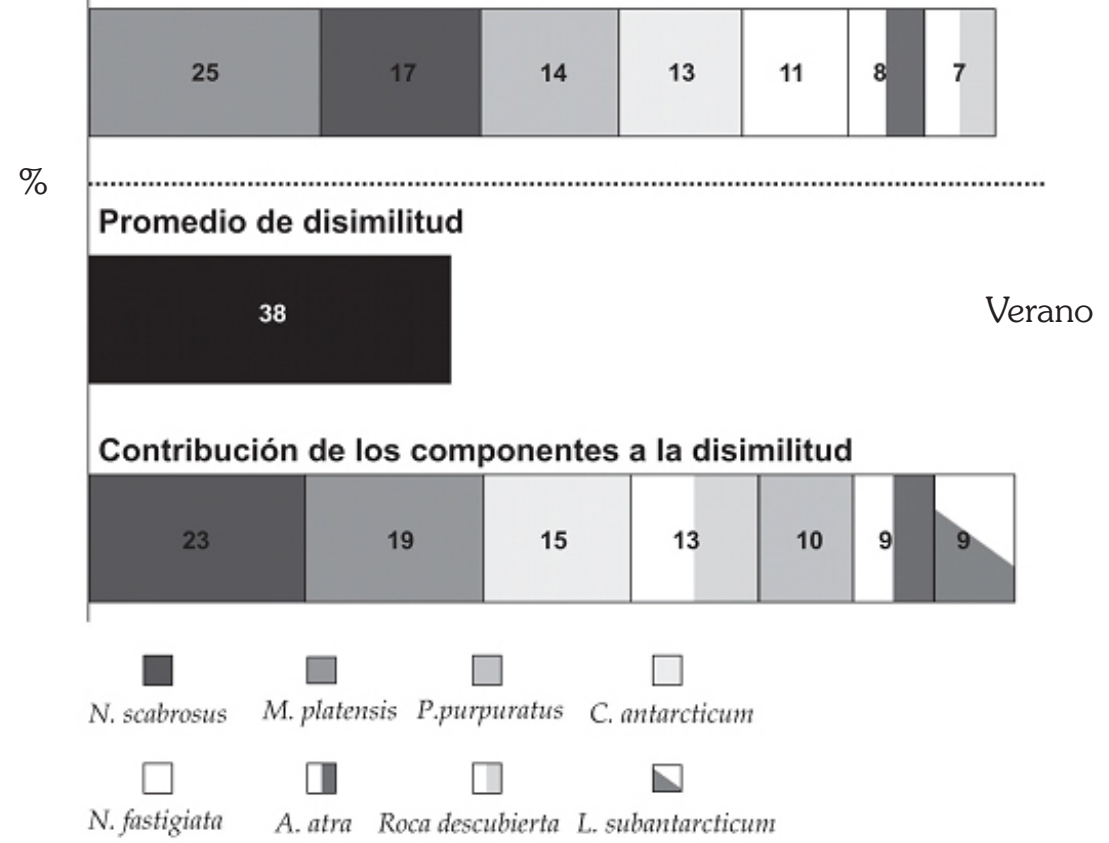

Fig. 2. Porcentaje promedio de disimilitud y porcentaje de contribución a la disimilitud por tipo de cobertura entre bolones y terrazas observados durante invierno y verano. 


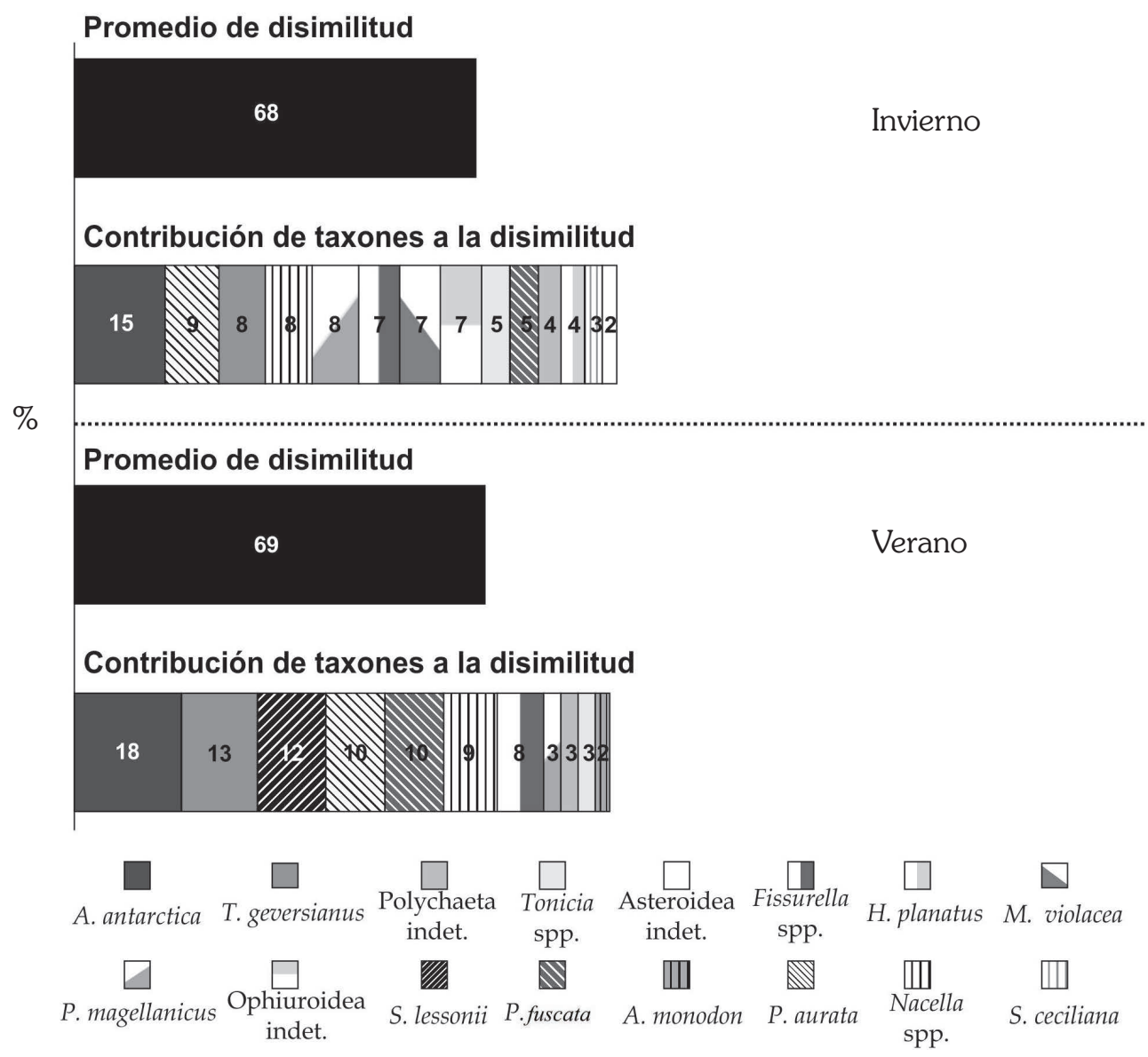

Fig. 3. Porcentaje promedio de disimilitud y porcentaje de contribución a la disimilitud por taxones móviles entre bolones y terrazas observados durante invierno y verano.

Mytilus platensis y Notochthamalus scabrosus (Fig. 2). Ambos taxones presentaron un mayor porcentaje de contribución en cobertura en plataformas. Por ejemplo, durante invierno $N$. scabrosus tuvo un $32,8 \%$ y M. platensis un 24,6 $\%$ de contribución a la cobertura en plataformas. Mientras que en verano, tuvo una mayor predominancia de roca descubierta (50,3\%), $M$. platensis (21,8 \%) y N. scabrosus (12,7 \%). Por otro lado, el sustrato de bolones presentó durante invierno una mayor cobertura de roca descubierta $(68,3 \%)$ y Caepidium antarcticum $(21,1 \%)$. Un patrón similar se observó en verano, esto porque el sustrato de bolones exhibió un alto porcentaje de cobertura de roca descubierta $(66,1 \%)$ y la presencia de Perumytilus purpuratus (17\%).

El análisis multivariado de homogeneidad de varianza PERMADISP no mostró diferencias significativas $(P>0,05)$ para taxones de hábitos móviles en sustrato de bolones y plataformas. No obstante, el análisis PERMANOVA que comparó la estructura comunitaria de taxones móviles entre bolones y plataformas mostró diferencias significativa $(P<0,05)$ en cada estación (verano $e$ invierno). El análisis SIMPER mostró que la disimilitud en la estructura comunitaria de taxones móviles entre ambos sustratos rocosos fue de un $68 \%$ en invierno y 69 \% verano (Fig. 3). Estas diferencias en invierno fueron principalmente explicadas por Anasterias antarctica, Plaxiphora aurata, Pseudechinus magellanicus y Trophon geversianus (Fig. 3). A su vez, durante verano estas diferencias fueron atribuidas a A. antarctica, T. geversianus, S. lessonii y P. aurata (Fig. 3). Por otra parte, al analizar la importancia de la contribución en abundancia por taxones móviles 


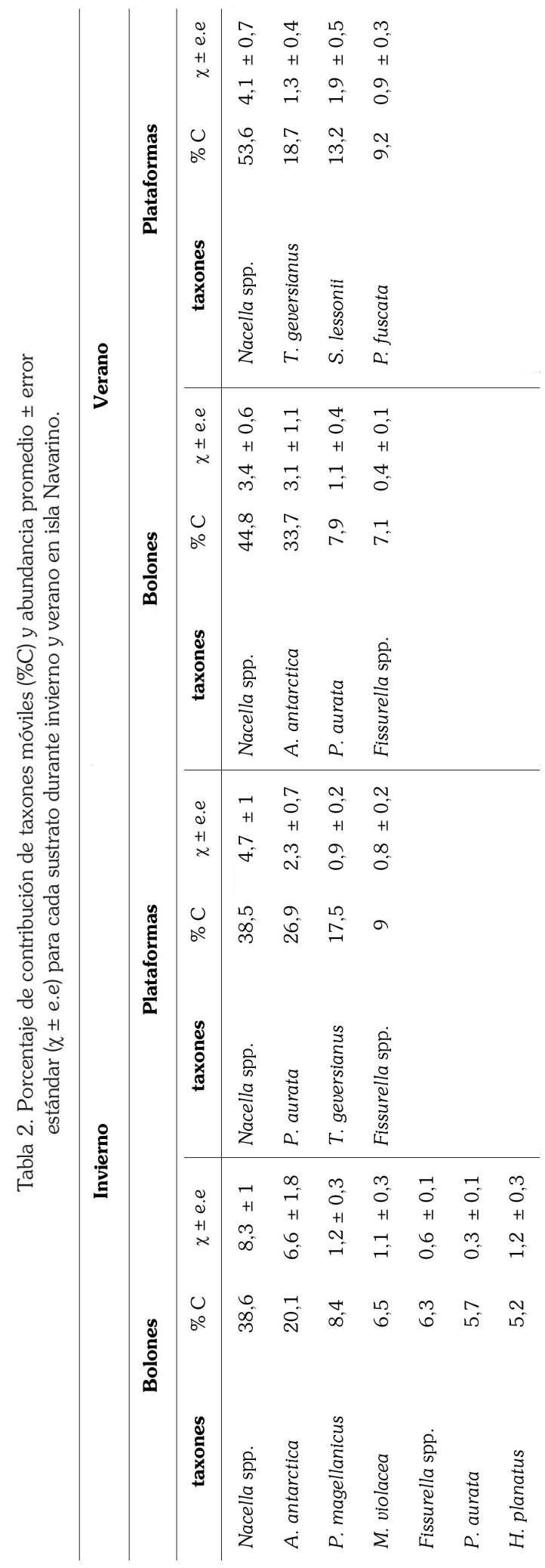

para cada sustrato, se observó que durante invierno Nacella spp., A. antarctica, P. magellanicus y Margarella violacea tuvieron una mayor abundancia y contribución al sustrato de bolones (Tabla 2). Mientras que, en esta misma estación, el sustrato de plataformas mostró una mayor contribución en la abundancia de Nacella spp., $P$. aurata, T. geversianus y Fissurella spp. (Tabla 2).

\section{DISCUSIÓN}

En altas latitudes en los canales y fiordos de Magallanes se ha observado que existe una alta heterogeneidad espacial (Benedetti-Cecchi \& Cinelli, 1997; Ríos et al. 2007; Ojeda et al. 2014). El presente estudio muestra atributos de esta heterogeneidad, la cual puede estar mediada por el tipo de sustrato intermareal, entre otras variables. Por ejemplo, nuestros datos muestran que el mayor porcentaje de cobertura de roca descubierta se observó en sustrato de bolones, mientras que un menor porcentaje se encontró en el sector de plataformas intermareales. Esto puede estar asociado a que el sustrato bolones es menos estable en comparación con una plataforma rocosa. Por lo tanto, presenta un mayor grado de perturbación por acción del oleaje, lo cual puede incrementarse con el viento oeste predominante en la zona del canal Beagle (Santana et al. 2006). De hecho, existen varios casos en donde un cierto grado de perturbación intermedia puede favorecer la riqueza de especies en playas de cantos rodados (e.g. Sousa, 1979). En este mismo sentido, un factor muy poco atendido en la estructura de la comunidades costeras de la ecorregión de Magallanes es el rol que juega el viento en asociación con los tipos de sustratos rocoso. En relación a esto, existe evidencia que en la Patagonia argentina el viento disminuye la humedad relativa en las costas rocosas, y por ende, aumenta la evaporación y el factor de estrés por desecación de las especies que habitan en el intermareal (Bertness et al. 2006). Concrétamente, registros específicos de velocidad del viento en la zona de bahía Róbalo muestran que costas con orientación oeste el viento puede alcanzar una velocidad promedio de $9,9 \mathrm{~m} / \mathrm{s}$, mientras que en costas con orientación norte $4,8 \mathrm{~m} / \mathrm{s}$ (Ojeda et al. 2014). Hipotéticamente, playas de bolones tienen a mayor cantidad de refugios, favoreciendo la conservación 
de la humedad y refugios termales (e.g. Gedan et al. 2011). No obstante, aún es necesario evaluar si factores acoplados como el viento y los tipos de sustrato (bolones vs. plataformas) pueden regular la estructura comunitaria en los canales y fiordos subantárticos de Magallanes.

En relación a nuestros registros, el intermareal de bolones presentó una mayor riqueza macroinvertebrados móviles, los cuales tienen una variedad de estructuras morfológicas externas. Por ejemplo, organismos que presentan concha (moluscos); esqueletos con placas calcáreas (equinodermos); con exoesqueleto con quitina (crustáceos) y finalmente con epidermis (peces) (e.g. Nacella ssp., A. antarctica, P. magallanicus, $H$. planatus y $H$. bispinis). Un contraste de aquello es el sustrato de plataforma, en donde existe casi exclusivamente predominancia de macroinvertebrados con concha (e.g. Nacella ssp., P. aurata, T. geversianus y $S$. lessonii). Estas diferencias en la estructura comunitaria y composición de especies evidencian que las características arquitectónicas de un sustrato pueden favorecer o no la presencia de un grupo de especies, la cual puede acentuarse en zonas templadas frías. Por ejemplo, se ha descrito que en altas latitudes la mayor diversidad de especies se encuentra bajo los bolones, debido la trimensionalidad de este sustrato intermareal (Waller, 2008). Finalmente, estos antecedentes refuerzan la noción que en sitios de importancia para la conservación como la zona costera del parque Etnobotánico Omora no sólo es importante evaluar la riqueza de especies y su abundancia, sino también los atributos funcionales de las especies que componen las comunidades (rasgos morfológicos, características tróficas o estrategias reproductivas). Al respecto, se ha documentado que estos mismos sitios intermareales de bolones de bahía Róbalo son importantes para la alimentación y postura de masas de huevos de peces del suborden Notothenioidei (ver Hüne \& Vega, 2015).

En la región de canales y fiordos subantárticos el macroherbívoro más abundantes en las costas rocosas corresponde a los patelogasterópodos del género Nacella spp. (Guzmán 1978; Ríos \& Gerdes, 1997; Rosenfeld et al. 2013; Ojeda et al. 2014). Según nuestros datos Nacella spp. es el taxón de hábito móvil con mayor abundancia, tanto en sustrato de bolones como plataformas.
Este grupo posee varios atributos para ser exitoso en amplio nicho ecológico en ambientes subantárticos. Uno de ellos está asociado a su mecanismo de alimentación sofisticado con una rádula docoglossa, la cual es una estructura compleja de dientes mineralizados. Esto les permite excavar y raspar la roca, permitiéndole acceder a oferta alimenticia de microalgas bentónicas, algunas especies macroalgas (e.g. Laminariales) y algas coralinaceas (Steneck \& Watling, 1982).

En este sentido, se ha descrito que las especies de Nacella en Magallanes pueden consumir perifiton (cianoficeas, diatomeas etc..), macroalgas (filamentosas, cilindricas y algunas corticadas) e invertebrados (e.g. foraminiferos micro-moluscos) (Rosenfeld et al. 2018).

En conclusión, el tipo de sustrato cumple un rol en los cambios de la composición de las especies, lo cual genera diferencias en la estructura de las comunidades de los intermareales próximos al Parque Etnobotánico Omora (sitio prioritario para la conservación). Se identificó que en bahía Róbalo el sustrato de bolones presentaba una mayor riqueza de taxones que el sustrato de plataformas. A su vez, en el sustrato de bolones se observó mayor cantidad de macroinvertebrados con diversos atributos funcionales, probablemente debido a que este hábitat provee mayor cantidad de refugios. Una proyección de esta investigación es que futuros estudios de la ecología intermareal subantártica deberían considerar la relevancia de los factores ambientales (e.g. intensidad del viento, orientación geográfica de las playas, y tamaño y tipo del sustrato rocoso) en la estructuración de las especies y la diversidad funcional de las comunidades subantárticas de Magallanes.

\section{AGRADECIMIENTOS}

Al apoyo logístico brindado por la Universidad de Magallanes sede Puerto Williams y al Instituto de Ecología y Biodiversidad (IEBCHILE). Se agradece al soporte administrativo de la Dirección de Investigación de la Universidad de Magallanes.

\section{LITERATURA CITADA}

Aldea, C., \& Rosenfeld, S. (2011). Moluscos intermareales de la Playa Buque Quemado 
(Estrecho de Magallanes, Chile). Revista de Biología Marina y Oceanografía, 46, 115124.

Anderson, M. J. (2001). A new method for nonparametric multivariate analysis of variance. Austral Ecology, 26(1), 32-46.

Anderson, M. J., Ellingsen, K. E., \& McArdle, B. H. (2006). Multivariate dispersion as a measure of beta diversity. Ecology Letters, 9(6), 683-693.

Banks, S. A., \& Skilleter, G. A. (2007). The importance of incorporating fine-scale habitat data into the design of an intertidal marine reserve system. Biological Conservation, 138(1), 13-29.

Benedetti-Cecchi, L., \& Cinelli, F. (1997). Spatial distribution of algae and invertebrates in the rocky intertidal zone of the Strait of Magellan: are patterns general? Polar Biology, 18(5), 337-343.

Benedetti-Cecchi, L., Bulleri, F., Acunto, S., \& Cinelli, F. (2001). Scales of variation in the effects of limpets on rocky shores in the northwest Mediterranean. Marine Ecology Progress Series, 209, 131-141.

Bertness, M. D., Crain, C. M., Silliman, B. R., Bazterrica, M. C., Reyna, M. V., Hidalgo, F., \& Farina, J. K. (2006). The community structure of Western Atlantic Patagonian rocky shores. Ecological Monographs, 76(3), 439-460.

Clarke, K. R., \& Gorley, R. N. (2006). PRIMER v6: User Manual/Tutorial. Plymouth: PRIMER-E.

Cruz-Motta, J. J., Miloslavich, P., Palomo, G., Iken, K., Konar, B., Pohle, G., Trott, T... \& Shirayama Y. (2010). Patterns of spatial variation of assemblages associated with intertidal rocky shores: a global perspective. PLoS ONE, 5, e14354. doi:10.1371/ journal.pone.0014354.

Dell, R. K. (1964). Antarctic and sub-Antarctic Mollusca: Amphineura, Scaphopoda and Bivalvia. Discovery Reports, 33, 99-250.

Dunmore, R. A., \& Schiel, D. R. (2003). Demography, competitive interactions and grazing effects of intertidal limpets in southern New Zealand. Journal of Experimental
Marine Biology and Ecology, 288(1), 1738.

Gedan, K. B., Bernhardt, J., Bertness, M. D., \& Leslie, H. M. (2011). Substrate size mediates thermal stress in the rocky intertidal. Ecology, 92(3), 576-582.

Gunnarsson, K., \& Ingolfsson, A. (1995). Seasonal changes in the abundance of intertidal algae in Southwestern Iceland. Botanica Marina, 38, 69-78.

Guzmán, L. (1978). Patrón de distribución espacial y densidad de Nacella magellanica (Gmelin, 1971) en el intermareal del sector oriental del Estrecho de Magallanes (Mollusca, Gastropoda). Anales del Instituto de la Patagonia, 9, 205-219.

Hervé, F., Quiroz D., \& Duhart, P. (2009) Principal Geological Aspects of the Chilean Fjord Region. In: V. Häussermann \& G. Försterra (Eds.), Fauna Marina Bentónica de la Patagonia Chilena (pp. 30-42). Santiago: Nature in Focus.

Hüne, M., \& Vega, R. (2016). Feeding habits in two sympatric species of Notothenioidei, Patagonotothen cornucola and Harpagifer bispinis, in the Chilean Patagonian channels and fjords. Polar Biology, doi 10.1007/ s00300-016-1892-3

Ojeda, J., Rosenfeld, S., Marambio, J., Rozzi, R., \& Mansilla, A. (2014). Patrones estacionales y espaciales de la diversidad de moluscos intermareales de bahía Róbalo, canal Beagle, Reserva de la Biosfera Cabo de Hornos, Chile. Revista de Biología Marina y Oceanografía, 49(3), 493-509.

Paine, R. T. (1980). Food webs: linkage, interaction strength and community infrastructure. Journal of Animal Ecology, 49(3), 667685.

Powell, A. W. B. (1951). Antarctic and Subantarctic Mollusca: Pelecypoda and Gastropoda. Discovery Reports, 26, 47-196.

Ramos, E., de Terán, J. R. D., Puente, A., \& Juanes, J. A. (2016). The role of geomorphology in the distribution of intertidal rocky macroalgae in the $\mathrm{NE}$ Atlantic region. Estuarine, Coastal and Shelf Science, 179, 90-98.

Reid, D. G., \& Osorio, C. (2000). The shallow- 
water marine mollusca of the Estero Elefantes and Laguna San Rafael, southern Chile. Bulletin of the Natural History Museum of London, Zoology, 66, 109-146.

Ríos, C., \& Gerdes, D. (1997). Ensamble bentónico epifaunístico de un campo intermareal de bloques y cantos en Bahía Laredo, Estrecho de Magallanes. Anales del Instituto de la Patagonia, 25, 47-55.

Ríos, C., Arntz, W. E., Gerdes, D., Mutschke, E., \& Montiel, A. (2007). Spatial and temporal variability of the benthic assemblages associated to the holdfasts of the kelp Macrocystis pyrifera in the Straits of Magellan, Chile. Polar Biology, 31(1), 89100.

Rosenfeld, S., Marambio, J., \& Aldea, C. (2013). Comparación de ensambles de moluscos en dos sustratos intermareales de la cuenca central del Estrecho de Magallanes intermareales de la cuenca central del Estrecho de Magallanes. Amici Molluscarum, 21(2), 7-18.

Rosenfeld, S., Marambio, J., Ojeda, J., Rodríguez, J.P., González-Wevar, C.A., Gerard, K., Contador, T.,... \& Mansilla, A. (2018). Trophic ecology of two coexisting Sub-Antarctic limpets of the genus Nacella: Spatio-temporal variation in food availability and diet composition of Nacella magellanica and N. deaurata in the SubAntarctic Ecoregion of Magellan. ZooKeys, (in press).

Rozzi, R., Anderson, C. B., Pizarro, J. C., Massardo, F., Medina, Y., Mansilla, A., Kennedy, J. H.,... \& Kalin, M. T. (2010). Filosofía ambiental de campo y conservación biocultural en el Parque Etnobotánico Omora: Aproximaciones metodológicas para ampliar los modos de integrar el componente social ("S") en Sitios de Estudios Socio-Ecológicos a Largo Plazo (SESELP). Revista Chilena de Historia Natural, 83, 27-68.

Rozzi, R., Armesto, J. J., Gutierrez, J., Massardo, F., Likens, G., Anderson, C. B., Poole, A., Moses, K.,... \& Kalin, M. T. (2012). Integrating ecology and environmental ethics: Earth stewardship in the southern end of the Americas. BioScience, 62, 226236.

Santana, A., Porter, C., Butorovic, N., \& Olave, C. (2006). Primeros antecedentes climatológicos de Estaciones Automáticas (AWS) en el canal Beagle, Magallanes, Chile. Anales del Instituto de la Patagonia, 34, 5-20.

Sherman, C. D., Peucker, A. J., \& Ayre, D. J. (2007). Do reproductive tactics vary with habitat heterogeneity in the intertidal sea anemone Actinia tenebrosa?. Journal of Experimental Marine Biology and Ecology, 340(2), 259-267.

Sirenko, B. (2006). Report on the present state of our knowledge with regard to the chitons (Mollusca: Polyplacophora) of the Magellan Strait and Falkland Islands. Venus, 65, 8189.

Soto, E. H., Báez, P., Ramírez, M. E., Letelier, S., Naretto, J., \& Rebolledo, A. (2012). Biotopos marinos intermareales entre Canal Trinidad y Canal Smyth, Sur de Chile. Revista de Biología Marina y Oceanografía, 47(2), 177-191.

Sousa, W. P. (1979). Disturbance in marine intertidal boulder fields: the nonequilibrium maintenance of species diversity. Ecology, 60(6), 1225-1239.

Spalding, M. D., Fox, H. E., Allen, G. R., Davidson, N., Ferdaña, Z. A., Finlayson, M., Halpern, B. S.,... \& Robertson, J. (2007). Marine ecoregions of the world: a bioregionalization of coastal and shelf areas. BioScience, 57(7), 573-583.

Steneck, R. S., \& Watling, L. (1982). Feeding capabilities and limitation of herbivorous molluscs: a functional group approach. Marine Biology, 68(3), 299-319.

Valdivia, N., Díaz, M. J., Holtheuer, J., Garrido, I., Huovinen, P., \& Gomez, I. (2014). Up, down, and all around: scale-dependent spatial variation in rocky-shore communities of Fildes Peninsula, King George Island, Antarctica. PloS ONE, 9(6), e100714.

Waller, C. L. (2008). Variability in intertidal communities along a latitudinal gradient in the Southern Ocean. Polar Biology, 31(7), 809-816. 
